\title{
Block Copolymers of Poly(2-oxazoline)s and Poly(meth)acrylates: A Crossover between Cationic Ring-Opening Polymerization (CROP) and Reversible Addition-Fragmentation Chain Transfer (RAFT)
}

Andreas Krieg, ${ }^{\dagger, \ddagger, \S}$ Christine Weber, ${ }^{\dagger, \ddagger}$ Richard Hoogenboom, $"$ C. Remzi Becer, ${ }^{*, \perp}$ and Ulrich S. Schubert $*, \dagger, \ddagger$

\author{
${ }^{\dagger}$ Laboratory of Organic and Macromolecular Chemistry (IOMC), Friedrich Schiller University of Jena, Humboldtstr. 10, 07743, Jena, \\ Germany \\ ${ }^{\ddagger}$ Jena Center for Soft Matter (JCSM), Friedrich Schiller University of Jena, Humboldtstr. 10, 07743, Jena, Germany \\ ${ }^{\S}$ Dutch Polymer Institute (DPI), John F. Kennedylaan 2, 5612AB, Eindhoven, The Netherlands \\ "Supramolecular Chemistry Group, Department of Organic Chemistry, Ghent University, Krijgslaan 281 S4, B-9000, Ghent, Belgium \\ ${ }^{\perp}$ Department of Chemistry, University of Warwick, Coventry CV4 7AL, United Kingdom
}

\section{Supporting Information}

ABSTRACT: To combine the advantages of poly(2-oxazoline)s $(\mathrm{POx})$ and controlled radical polymerization (CRP) techniques, the cationic ring-opening polymerization (CROP) of 2-methyl-2oxazoline (MeOx), 2-ethyl-2-oxazoline (EtOx), and 2-n-nonyl-2oxazoline (NonOx) were terminated with a carboxylic acid functionalized chain transfer agent (CTA). The obtained PEtOx with a high degree of functionalization (DF) was used as macroCTA for the reversible addition-fragmentation chain transfer (RAFT) polymerization of various vinylic monomers (styrene $(\mathrm{St})$, methyl acrylate (MA), tert-butyl acrylate $(t \mathrm{BuA})$, acrylic acid (AA), N,N-dimethyl acrylamide (DMAAm), and N,N-dimethylaminoethylacrylate (DMAEA)) resulting in well-defined block copolymers. Thus, in this work we report a versatile route to combine poly(2-oxazoline)s prepared via CROP with RAFT polymerization for the synthesis of novel block copolymers.
M odern drug delivery concepts often include polymeric drug carriers that contain poly(ethylene glycol) (PEG) chains due to their solubility in water and stealth behavior; that is, they are not easily recognized by the immune systems. ${ }^{1}$ However, in a minority of cases PEG has been shown to interact with the immune system, degrade under stress, and accumulate in the body. ${ }^{2}$ For these reasons the scientific community is continuously searching for potential alternatives that can replace PEGylated systems. Poly(2-oxazoline)s (POx) are one of the promising candidates for this challenge. ${ }^{3}$ The hydrophilicity of the well-defined polymers can easily be varied by utilization of a range of 2-oxazoline monomers with different substituents in 2-position of the ring. ${ }^{4}$ In addition, the nature of the living cationic ring-opening polymerization (CROP), ${ }^{5}$ which is used for the preparation of POx, offers an advantage to end-functionalize polymer chains with a large range of functional groups. On the other hand, the cationic polymerization mechanism does not tolerate monomers containing nucleophilic or proton donating functional groups and, therefore, prohibits direct access to certain properties, such as $\mathrm{pH}$ responsiveness ${ }^{6}$ or the ability to complex DNA. ${ }^{7}$ However, such moieties are well-tolerated by radical polymerization techniques. Thus, the combination of controlled radical

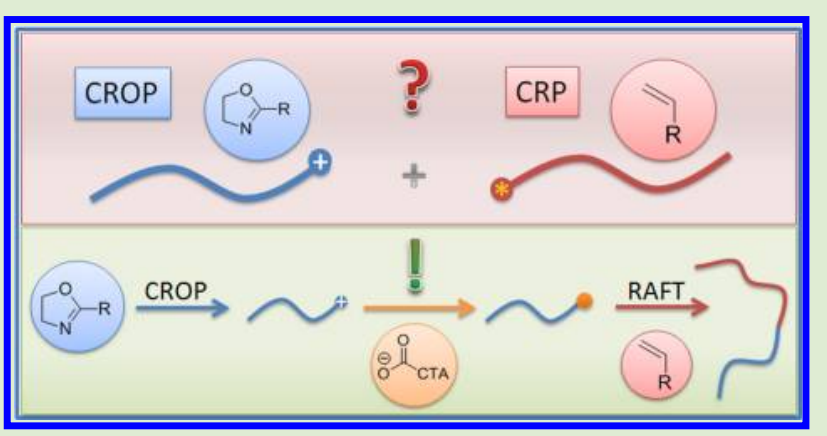

polymerization (CRP) with CROP should allow the combination of interesting properties, which are only accessible by each specific polymerization method. The first example toward 2oxazoline based block copolymers was reported based on the combination of CROP and atom transfer radical polymerization (ATRP), ${ }^{8}$ but the necessity of copper is not desirable with respect to the application of block copolymers in biological systems. More recently, the combination of CROP and nitroxide mediated polymerization (NMP) was demonstrated, which is, however, limited to styrenic and acrylate monomers. Another widely employed CRP technique, which does not require any metal catalyst and is applicable to nearly all vinyl monomers, is the reversible addition-fragmentation chain transfer (RAFT) polymerization. ${ }^{9}$ The combination of CROP and RAFT has been reported for poly(thiourethane) and poly(THF) based block copolymers. ${ }^{10}$ The synthesis of the macro chain transfer agents (macro-CTAs) was carried out via end-capping with an appropriate CTA-ion in the first case or a

Received: March 18, 2012

Accepted: May 25, 2012

Published: June 8, 2012 
reaction involving 2-steps yielding a CTA functionalized dendrimer in the latter one. A combination of 2-oxazolines and RAFT, which has only been applied within the synthesis of comb polymers up to now, ${ }^{11}$ would deliver a perfect tool for the straightforward synthesis of block copolymers that possess both the advantages of $\mathrm{POx}$ and a wide range of polymers derived from vinylic monomers, such as acrylates, acrylamides, or styrene.

As shown in Scheme 1, the cationic oxazolinium species from the CROP can be directly end functionalized by addition of a RAFT agent that bears a deprotonated carboxylic acid functionality.

Scheme 1. Schematic Representation of the Synthesis Route towards Block Copolymers Composed of 2-Oxazolines and Vinyl Monomers

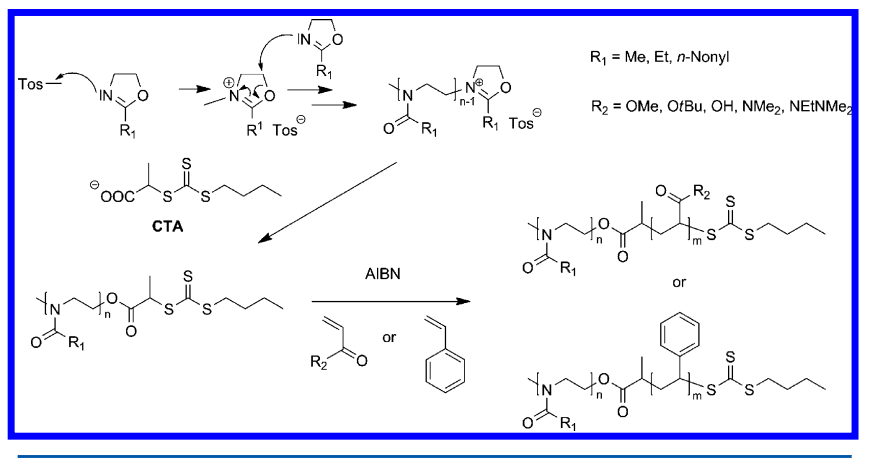

Subsequently, the resulting POx based RAFT agents are used as macro chain transfer agents (CTAs) for the CRP of the second block.

To synthesize POx based CTAs, three different 2-oxazoline monomers, namely, 2-methyl-2-oxazoline ( $\mathrm{MeOx})$, 2-ethyl-2oxazoline (EtOx), and 2-n-nonyl-2-oxazoline ( $\mathrm{NonOx}$ ), were polymerized under microwave irradiation at $140{ }^{\circ} \mathrm{C}$ using methyl tosylate as an initiator. Since the CROP of 2-oxazolines is a living polymerization process, the degree of polymerization can easily be controlled by varying the ratio of monomer to initiator $([\mathrm{M}] /[\mathrm{I}])$. To facilitate a reasonable determination of the polymer end groups by NMR spectroscopy as well as mass spectrometry, the $[\mathrm{M}] /[\mathrm{I}]$ ratio was chosen to be rather low (10 or 20). Further advantages of the livingness of the CROP are the possibility to perform the polymerization until full conversion of monomer and the possibility to directly end functionalize the living cationic oxazolinium chain end by the attack of suitable nucleophiles. In earlier studies, carboxylic acids, that were deprotonated in situ by addition of triethylamine as a base, have been shown to enable very high degrees of functionalization. ${ }^{11}$ Therefore, a trithiocarbonate based RAFT agent bearing a carboxylic acid function, namely, 2(butylthiocarbonothioylthio) propanoic acid, was applied for the end functionalization of the living poly(2-oxazoline) chains. The resulting macro-CTAs were characterized by size exclusion chromatography (SEC), ${ }^{1} \mathrm{H}$ NMR spectroscopy, and matrixassisted laser desorption-ionization (MALDI) as well as electrospray ionization (ESI) time-of-flight mass spectrometry (TOF MS), as summarized in Table 1.

As demonstrated by SEC, the livingness of the CROP resulted in narrow molar mass distributions with PDI values below 1.26. However, with respect to the application of the $\mathrm{POx}$ as macro chain transfer agents, a sufficiently high degree of functionalization (DF) of the polymer $\omega$ end groups is of even
Table 1. Characterization Data of the Synthesized Poly(2oxazoline) Macro Chain Transfer Agents

$\begin{array}{lcccccc} & \mathrm{R}_{1} & \mathrm{M} / \mathrm{I} & \mathrm{DP}^{d} & \begin{array}{c}M_{\mathrm{n}}(\mathrm{SEC})^{a} \\ \left(\mathrm{~g} \mathrm{~mol}^{-1}\right)\end{array} & \begin{array}{c}\mathrm{PDI} \\ (\mathrm{SEC})^{a}\end{array} & \begin{array}{c}\mathrm{DF} \\ (\mathrm{NMR})^{c}\end{array} \\ \text { P1 } & \mathrm{Et} & 10 & 11 & 560 & 1.26 & \text { quant } \\ \text { P2 } & \mathrm{Et} & 20 & 22 & 2660 & 1.09 & \text { quant } \\ \text { P3 } & \mathrm{Me} & 21 & 21 & 3170^{b} & 1.13^{b} & 73 \% \\ \text { P4 } & \text { Non } & 19 & 21 & 4300 & 1.13 & 95 \%\end{array}$

${ }^{a}$ Obtained from SEC $\left(\mathrm{CHCl}_{3}\right.$, PS calibration). ${ }^{b}$ Obtained from SEC (DMA, PS calibration). ${ }^{c}$ Calculated by comparison of integral values of peaks derived from $\alpha$ - and $\omega$-end groups. ${ }^{d}$ Calculated from initiator and polymer backbone signals in the ${ }^{1} \mathrm{H}$ NMR spectrum.

greater importance. An opportunity to obtain quantitative information (within borders of the applied method) about the DF is the integration of the signals in the ${ }^{1} \mathrm{H}$ NMR spectra that are derived from the polymer backbone and the end groups (Figure S1, Supporting Information (SI)). Hereby, the absence of CTA that is not connected to the POx was ensured by purification of the macro-CTAs using preparative SEC. Assuming a negligible amount of chain transfer during the CROP, the DP of the macro-CTAs can be calculated from the peak integral of the initiating methyl group at the $\alpha$ chain end of the polymer $(3.0 \mathrm{ppm})$ and the peak integral of the polymer backbone (3.2-3.7 ppm). Indeed, the obtained values coincide well with the used $[\mathrm{M}] /[\mathrm{I}]$ ratio and, therefore, hint at near quantitative functionalization at the $\alpha$ chain end. With that knowledge, the DF with CTA at the $\omega$ chain end can be estimated by comparison of the peak integrals derived from $\alpha$ and $\omega$-end groups. The $\omega$ terminal methyl peaks (0.8 ppm) were well enough separated from the polymer side chain signals to be used for this purpose. Additionally, the integration of the methine proton peak of the CTA (4.7 ppm) resulted in similar high DF values (mostly $>85 \%$ ).

Soft ionization mass spectrometric techniques, such as MALDI and ESI-TOF MS, nowadays offer a good opportunity for the end group determination of well-defined polymers, although they do not provide quantitative information about the ratio of various end groups that might be present. ${ }^{12}$ As shown in Figure 1 for P2, the mass spectra obtained by MALDI and ESI-TOF MS show the same main distribution, which can be assigned to PEtOx that is ionized with a sodium cation and

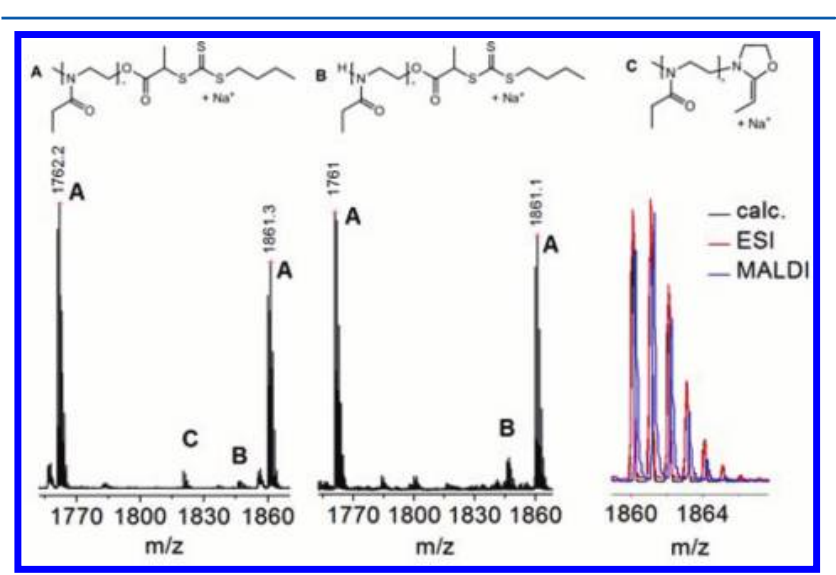

Figure 1. Magnified portion of the mass spectra of the PEtOx macroCTA P2 and assignment of the observed distributions. Left: MALDITOF mass spectrum. Center: ESI-TOF mass spectrum. Right: Overlay of calculated and measured isotopic patterns of distribution A. 
that is carrying both a methyl and a CTA end group. A minor distribution reveals the presence of proton initiated POx chains (a side product resulting from chain transfer reactions during the CROP). However, also the proton initiated chains are functionalized with the desired CTA. The harsher ionization of the macro-CTA during the MALDI process additionally results in a fragmentation of the trithiocarbonate based end group, which is connected to the POx via an ester bond. Further evidence for the fragmentation is provided by the MALDI-TOF tandem MS spectra (Figure S2, SI), where exactly the same fragmentation occurs when a parent ion from the main distribution is chosen for MS MS analysis.

The synthesis of block copolymers was achieved by utilization of the synthesized macro-CTAs in the RAFT polymerization using 2,2'-azobis(isobutyronitrile) (AIBN) as the initiator. Thereby the growth of the second block takes place at the $\omega$ chain end of the poly(2-oxazoline) and results in a block copolymer bearing an ester bond as linkage between both blocks. The trithiocarbonate-based PEtOx macro-CTAs proved suitable for the synthesis of block copolymers employing multiple vinylic monomers, such as styrene (St), acrylic acid (AA), dimethyl acrylamide (DMAAm), and various acrylates: methyl acrylate (MA), tert-butyl acrylate $(t-\mathrm{BuA})$, and $N, N$-dimethylaminoethylacrylate (DMAEA). The characterization data of the resulting block copolymers by means of SEC and ${ }^{1} \mathrm{H}$ NMR spectroscopy are presented in Table 2.

Table 2. Characterization Data of the Synthesized Block Copolymers Using P2 as a Macro-CTA

\begin{tabular}{llcccc} 
& \multicolumn{1}{c}{$\mathrm{R}_{2}$} & M/CTA & $\begin{array}{c}M_{\mathrm{n}}(\mathrm{SEC})^{a} \\
\left(\mathrm{~g} \mathrm{~mol}^{-1}\right)\end{array}$ & $\begin{array}{c}\text { PDI } \\
(\mathrm{SEC})^{a}\end{array}$ & $\begin{array}{c}\mathrm{M}_{1}: \mathrm{M}_{2} \\
\left({ }^{1} \mathrm{H} \mathrm{NMR}\right)\end{array}$ \\
B1 & St & 100 & 3600 & 1.19 & $22: 9$ \\
B2 & $t$-BuA & 100 & 11000 & 1.18 & $22: 112$ \\
B3 & AA & 25 & $6500^{b}$ & $1.40^{b}$ & $22: 27$ \\
B4 & DMAAm & 100 & 17000 & 1.11 & $22: 113$ \\
B5 & DMAEA & 25 & 5400 & 1.13 & $22: 19$ \\
B6 & MA & 100 & 5200 & 1.25 & $22: 29$
\end{tabular}

${ }^{a}$ Obtained from SEC $\left(\mathrm{CHCl}_{3}\right.$, PS calibration). ${ }^{b}$ Obtained from SEC (DMA, PS calibration).

The SEC traces of the obtained precipitated block copolymers are presented in Figure 2 (the SEC trace of B3 in DMA is shown in Figure S3 of the SI), revealing monomodal peaks and narrow molar mass distributions with PDI values mostly below 1.3, as typically observed for RAFT polymer-

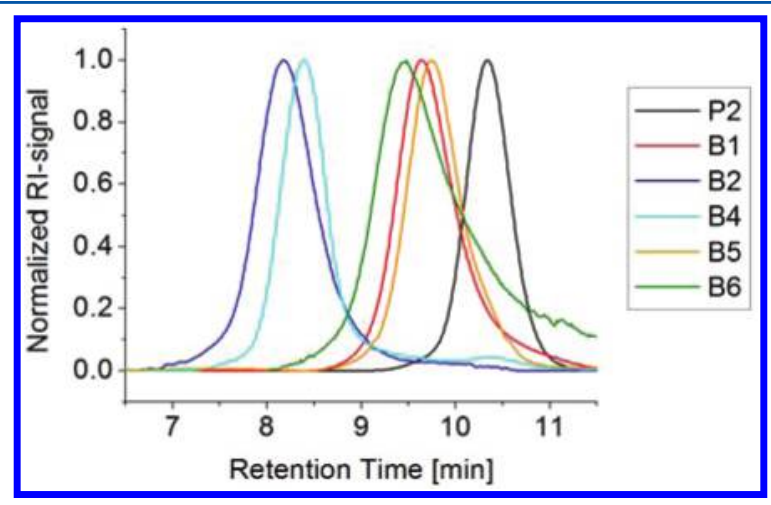

Figure 2. Overlay of SEC traces $\left(\mathrm{CHCl}_{3}\right)$ of the synthesized block copolymers and utilized macro-CTA. ization. The observed shift of the block copolymer SEC traces in comparison to $\mathbf{P 2}$ demonstrates the effectiveness of the functionalized POx as macro-CTA. The rather small DP of the second block in B1, B5, and B6 results in a partial overlapping SEC trace with the macromonomer, but it is clearly shifted to lower retention time, too. Due to the principle of SEC measurements (via RI-detector) with the intrinsic overestimation of high molar mass fractions, it is difficult to estimate the chain extension efficiency. Nevertheless, the amount of remaining starting material relative to the obtained block copolymers can be stated as rather low, and the molar mass distributions derived from SEC analysis are presented in the SI. However, it should be clearly stated that they are not representative of the $M_{\mathrm{n}}$ values due to the fact that they are obtained from calibration with PS standards, which most likely have a different hydrodynamic volume than the prepared block copolymers.

The final composition of the purified block copolymers was determined by ${ }^{1} \mathrm{H}$ NMR spectroscopy (Figure 3), clearly

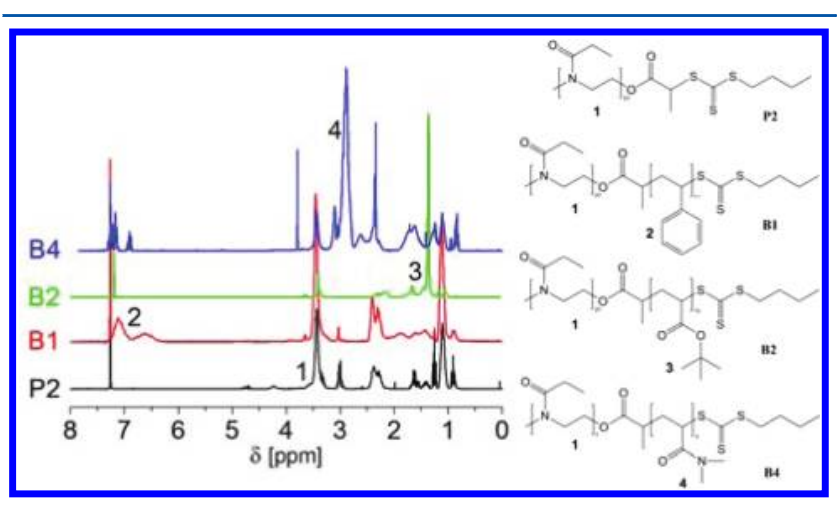

Figure 3. Exemplary ${ }^{1} \mathrm{H}$ NMR spectra $\left(\mathrm{CDCl}_{3}, 300 \mathrm{MHz}\right)$ of the purified block copolymers and the utilized PEtOx macro-CTA.

showing specific signals of both PEtOx as well as the vinylic blocks. In all spectra the peak derived from the POx backbone around $3.5 \mathrm{ppm}$ was sufficiently separated to permit a distinct integration and, thus, to determine the ratio of EtOx and vinylic monomers in the block copolymers. The utilized peaks are indicated in Figure 3 and derived from aromatic protons of PS for B1, the tert-butyl group of B2, and the methyl protons of the tertiary amide for B4. Assuming a DP of 22 of the PEtOx block resulted in the DP of the vinylic blocks that are given in Table 2.

The small DP of the PS block of B1 resulted in a block copolymer with a sufficiently low molar mass to enable a detailed characterization via MALDI-TOF MS (Figure 4).

The mass spectrum shows two main distributions, derived from PEtOx- $b$-PS and PEtOx homopolymer. The P(EtOx) distribution (B) is identical with the observed nonfunctionalized species $\mathrm{C}$ shown in Figure 1 and is most likely formed by cleavage of the ester unit that connects both blocks during the MALDI measurement (in a similar fashion as could already be observed in the MALDI-TOF MS of the macro-CTA). However, it cannot be excluded that traces of PEtOx homopolymer may be present in B1. But the fact that no PEtOx species bearing the CTA end group can be observed in the MALDI-TOF mass spectrum indicates a high chain extension efficiency. The PEtOx-b-PS distribution (A) shows multiple peaks with an $m / z$ distance of $5\left(\mathrm{M}_{\mathrm{S}}-\mathrm{M}_{\mathrm{EtOx}}\right)$ 


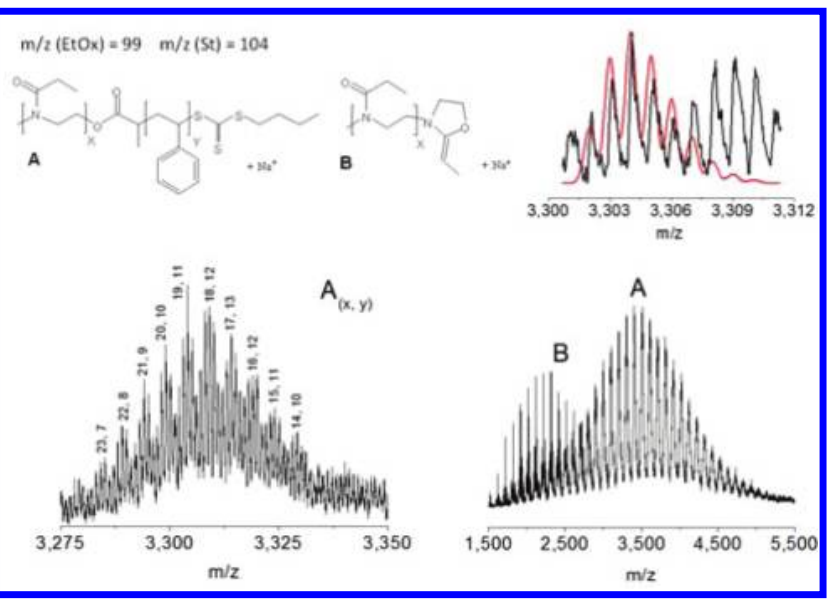

Figure 4. MALDI-TOF/MS spectrum of $\mathrm{P}(\mathrm{EtOx}-b-\mathrm{St})$ (bottom right) with magnification (bottom left), schematic representations of the assigned structures (top left), and a comparison of calculated (red line) and measured (black line) isotopic patterns for $\mathrm{P}\left(\mathrm{EtOx}_{19}-b-\mathrm{St}_{11}\right)$ $\left(\mathrm{A}_{(19,11)}\right)$ (top right).

reflecting the DP combinations of both included monomers given in Figure 4.

In conclusion, the reported synthetic approach enabled the easy and straightforward synthesis of a wide range of block copolymers consisting of blocks that are accessible only by the application of different polymerization techniques. Trithiocarbonate-based POx macro-CTAs were obtained by direct end functionalization after CROP in high DF and were applicable for RAFT polymerization of a broad range of vinylic monomers. In the obtained polymers advantages of both polymer types can be combined and, therefore, may lead to novel material properties for biological applications, which will be in the focus of our future research.

\section{ASSOCIATED CONTENT}

\section{S Supporting Information}

Experimental part, ${ }^{1} \mathrm{H}$ NMR spectra, full MALDI-TOF MS of P2, magnification of MALDI-TOF MS of B1, and MALDITOF MS MS of PEtOx macroinitiators. This material is available free of charge via the Internet at http://pubs.acs.org.

\section{AUTHOR INFORMATION}

\section{Corresponding Author}

*E-mail: C.R.Becer@warwick.ac.uk; Ulrich.Schubert@uni-jena. de.

\section{Notes}

The authors declare no competing financial interest.

\section{ACKNOWLEDGMENTS}

The authors thank the Dutch Polymer Institute (DPI, technology area HTE) for financial support. C.R.B. is grateful for the funding from Science City Advantage West Midlands. R.H. thanks the European Union for financial support via a Marie Curie Career Integration Grant. The authors thank Anja Baumgärtel for performing the MALDI-TOF/MS and Esra Altuntas for the ESI-MS measurements.

\section{REFERENCES}

(1) Pasut, G.; Veronese, F. M. Prog. Polym. Sci. 2011, 32, 933-961.

(2) (a) Barz, M.; Luxenhofer, R.; Zentel, R.; Vicent, M. J. Polym. Chem. 2011, 2. (b) Knop, K.; Hoogenboom, R.; Fischer, D.; Schubert,
U. S. Angew. Chem., Int. Ed. 2010, 49, 6288-6308. (c) Marx, L.; Volet, G.; Amiel, C. J. Polym. Sci., Part A: Polym. Chem. 2011, 49, 4785-4793. (3) (a) Adams, N.; Schubert, U. S. Adv. Drug Delivery Rev. 2007, 59, 1504-1520. (b) Makino, A.; Kobayashi, S. J. Polym. Sci., Part A: Polym. Chem. 2010, 48, 1251-1270. (c) Schlaad, H.; Diehl, C.; Gress, A.; Meyer, M.; Demirel, A. L.; Nur, Y.; Bertin, A. Macromol. Rapid Commun. 2010, 31, 511-525. (d) Hoogenboom, R. Angew. Chem., Int. Ed. 2009, 48, 7978-7994.

(4) (a) Wiesbrock, F.; Hoogenboom, R.; Leenen, M. A. M.; Meier, M. A. R.; Schubert, U. S. Macromolecules 2005, 38, 5025-5034. (b) Hoogenboom, R.; Wiesbrock, F.; Leenen, M. A. M.; Meier, M. A. R.; Schubert, U. S. J. Comb. Chem. 2005, 7, 10-13.

(5) (a) Kagiya, T.; Narisawa, S.; Maeda, T.; Fukui, K. J. Polym. Sci., Polym. Lett. 1966, 4, 441-445. (b) Seeliger, W.; Aufderha., E; Diepers, W.; Feinauer, R.; Nehring, R.; Thier, W.; Hellmann, H. Angew. Chem., Int. Ed. 1966, 5, 875-888. (c) Tomalia, D. A.; Sheetz, D. P. J. Polym. Sci., Part A: Polym. Chem. 1966, 4, 2253-2265. (d) Levy, A.; Litt, M. J. Polym. Sci., Polym. Lett. 1967, 5, 881-886.

(6) Dai, S.; Ravi, P.; Tam, K. C. Soft Matter 2008, 4, 435-449.

(7) York, A. W.; Zhang, Y. L.; Holley, A. C.; Guo, Y. L.; Huang, F. Q.; McCormick, C. L. Biomacromolecules 2009, 10, 936-943.

(8) Becer, C. R.; Paulus, R. M.; Hoppener, S.; Hoogenboom, R.; Fustin, C. A.; Gohy, J. F. O.; Schubert, U. S. Macromolecules 2008, 41, $5210-5215$.

(9) (a) Moad, G.; Rizzardo, E.; Thang, S. H. Aust. J. Chem. 2006, 59, 669-692. (b) Moad, G.; Rizzardo, E.; Thang, S. H. Aust. J. Chem. 2009, 62, 1402-1472. (c) Moad, G.; Rizzardo, E.; Thang, S. H. Polymer 2008, 49, 1079-1131. (d) Barner-Kowollik, C.; Perrier, S. J. Polym. Sci., Part A: Polym. Chem. 2008, 46, 5715-5723.

(10) (a) Zhong, L.; Zhou, Y. F.; Yan, D. Y.; Pan, C. Y. Macromol. Rapid Commun. 2008, 29, 1385-1391. (b) Nagai, A.; Hamaguchi, T.; Kikukawa, K.; Kawamoto, E.; Endo, T. Macromolecules 2007, 40, 6454-6456.

(11) (a) Weber, C.; Becer, C. R.; Guenther, W.; Hoogenboom, R; Schubert, U. S. Macromolecules 2010, 43, 160-167. (b) Weber, C.; Becer, C. R.; Hoogenboom, R.; Schubertt, U. S. Macromolecules 2009, 42, 2965-2971.

(12) (a) Knop, K.; Jahn, B. O.; Hager, M. D.; Crecelius, A.; Gottschaldt, M.; Schubert, U. S. Macromol. Chem. Phys. 2010, 211, 677-684. (b) Hart-Smith, G.; Barner-Kowollik, C. Macromol. Chem. Phys. 2010, 211, 1507-1529. (c) Song, J. K.; van Velde, J. W.; Vertommen, L. L. T.; Smith, D. F.; Heeren, R. M. A.; van den Brink, O. F. Macromolecules 2011, 44, 1319-1326. (d) Ladaviere, C.; LacroixDesmazes, P.; Delolme, F. Macromolecules 2009, 42, 70-84. (e) Gruendling, T.; Weidner, S.; Falkenhagen, J.; Barner-Kowollik, C. Polym. Chem. 2010, 1, 599-617. 